\title{
The view of classroom light design optimization simulation
}

\author{
Shijie Wang \\ School of Electrical \& Electronic Engineering,North China Electric Power University, Baoding, \\ 071003 China \\ 328799219@qq.com
}

Keywords: optical illumination;environmental protection and energy saving;simulation;

\begin{abstract}
In view of the issue that present illumination facilities in classrooms can cause the waste of electric energy, this project group tried to solve the problem by designing the automatic light control system. The group started with the study of the electricity consumption of those lamps in the classroom and, with the help of MATLAB and LINGO, the established classroom model was analyzed, offering us the differential analysis and the data fitting of the light source coordinates. And software for the design of illumination, DIALux was adopted to implement the simulation test on the illumination of the classroom. We found the problem that the light distribution is uneven under the traditional configuration mode and put forward the relatively efficient way to arrange the distribution of lamps. Meeting the CIE standard, the configuration mode of the lamps increased the effective lighting area of the classroom and increased the efficiency of the electricity used for illumination. It has a broad prospect in the applied fields. And it can also help build a conservation-minded campus and achieve the goal of energy conservation and emission reduction.
\end{abstract}

\section{Introduction}

In order to respond to the call of "build a resource-efficient campus" from the Ministry of Education, many universities in our country have carried out the work of energy saving work. However, due to the insufficient research on the link of electricity using, the effective energy-conservation measures and related actions have not been formed. The waste of electricity using is still quite serious. After the research, the group found that the consumption of single lamp for illumination is not quite much, but because of the large number of lamps and the long period of using, its proportion in the entire electricity consumption of the school stays at a high level (about $40 \%-45 \%$ of the total electricity consumption) ${ }^{[1]}$.

This project group carries on the simulation experiment with the aid of DIALux, and put forward an even more energy-efficient scheme for the arrangement of lamps. Meanwhile the group designed the automatic control system to control the switch of lamps in classroom; thus the waste of electricity is effectively reduced. If all universities in the country adopt this plan, according to our estimate, the nation will save quite a large amount of electrical energy: if electricity costs 0.573 Yuan a kilowatt-hour, throughout the year all universities will reduce their expenditure of 0.625 billion Yuan, which will bring great social and the economic benefits. Meanwhile, through the energy-saving work developed in the universities, students' awareness of conservation will be reinforced and good habit of energy-saving will be formed, which will make them become the main force of building the resource-efficient society.

\section{Simulation experiment and its design}

\section{The design and its research plan}

\section{1) The current situation of the classroom illumination model}

The model adopted by the simulation experiment is from the North China Electric Power University (Baoding). The university gives its priority to the open management mode: the students here have no fixed class classrooms and no fixed seats; however, the administrators of the building are only responsible for the cleanness in it and students' security. The situation above causes the 
phenomenon of wasting: the lights keep glowing even if there is sufficient sunlight; the lights will not be turned off even if there is no one in the classroom at night; all of the lights are turned on even if few students are in the classroom. According to the "Standards for illumination design in building" (GB - 50034-2004) in China, the lighting power density in the classroom should not be greater than $11 \mathrm{w} / \mathrm{m}^{2}$ and the luminance should not be less than $300 \mathrm{~lx}{ }^{[2]}$ According to the internationally prevailed CIE standard, luminance should not be less than $500 \mathrm{~lx}$ (this simulation experiment is based on $500 \mathrm{~lx}$ standard). The lighting power density and intensity of illumination of the present model almost achieve the requirements of the CIE standard; however, the distribution of light is uneven. As a result, the effective lighting area, which actually meets the requirement of the intensity of illumination, is much narrower. Meanwhile, the number of students in the classroom at present is smaller than the most reasonable one, which is not conducive to make full use of the space of classroom.

\section{2) Statistics of the classroom model}

The parameters of the classroom and the desk: the size of the classroom is $7.0 \mathrm{~m} \times 7.0 \mathrm{mx} 3.5 \mathrm{~m}$; the height of lamps is $2.81 \mathrm{~m}$; the size of the small desks is $1.326 \mathrm{~m} \times 0.448 \mathrm{~m} \times 0.760 \mathrm{~m}$, the size of large desk is $2.078 \mathrm{~m} \times 0.448 \mathrm{~m} \times 0.760 \mathrm{~m}$.

Considering the factors that have influence on the actual lighting situation in the classroom and the advantage that DIALux simulation can comprehensively simulate these factors, the project group got the reflection coefficient of each part of the classroom as the table 1 shows.

Tab.1 Plane reflection coefficient in the classroom

\begin{tabular}{c|c}
\hline surface & reflection coefficient(\%) \\
\hline the floor & 25 \\
the front wall & 55 \\
the ceiling & 70 \\
the working surface & 42 \\
the back wall and the side wall & 75 \\
\hline
\end{tabular}

Through the survey of the lamp models that are commonly used in the classroom, the project group chose the model, 2 XTL - D36W.

The formula of average illuminance ${ }^{[3]}$ :

$E=\frac{\Phi \cdot N \cdot U \cdot K}{A}$

Among them:

$\Phi$ - the amount of the passed light, unit: lm;

$\mathrm{N}$ - the number of lamps;

$\mathrm{U}$ - the utilization coefficient of lamps;

$\mathrm{K}$ - maintenance coefficient;

A - the area of the classroom, unit: $\mathrm{m}^{2}$.

The uniformity of illuminance $(\mathrm{U} 0<1)$ formula:

$U_{0}=\frac{E_{\min }}{E_{a v}}$

Among them, Emin - minimum illumination;

Eav - average illuminance.

The higher the uniformity of illuminance is, the evener the distribution of light is and the more comfortable the eyes feel. On the contrary, the smaller it is, the more visual fatigue occurs. Therefore, under the circumstance that the average illuminance meets the CIE standard, we evaluated the advantages and disadvantages of the configuration mode according to the uniformity of illuminance. 


\section{The simulation scheme and result analysis}

\section{1) The simulation scheme}

After doing quite amount of research with the process of analyzing and considering, our group preceded the experiment to test the practical effect of the 3 schemes: the even layout, the cellular layout and the elliptic layout. Under the premise that lighting parameters meet the requirements of the CIE standard, the group got the coordinates respectively when the tubes in the classroom reached the highest point of EAV by interpolation analysis and data fitting with the aid of the software, MATLAB and LINGO.

(1) Ordinary configuration

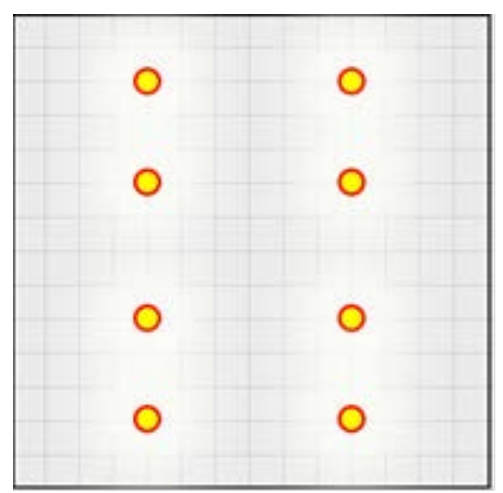

Fig.1 Lamps are evenly arranged

Lamps are evenly arranged as the chart 1 shows. At the very beginning of the experiment, our group preliminarily simulated this kind of arrangement according to the MATLAB, and then with the aid of LINGO, we analyzed and calculated the specific coordinates of the light by interpolation analysis when it reaches the highest uniformity of illuminance. After that, we got the intensity contour map and graphic model of the theoretical optimum light density under the coordinate.

The intensity contour map is showed in chart 2.

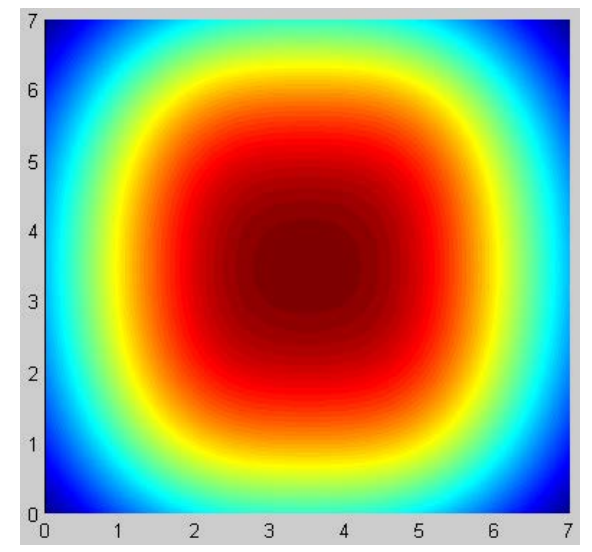

Fig.2 Scheme 1 corresponding intensity contour map

Our group considered the other influencing factors of lighting in the reality, such as the reflection coefficient of the wall and other materials, and then the simulation of the lighting outcome in the real illumination places was carried out, whose result showed that the software meets all the requirements for illumination design. ${ }^{[4-5]}$

Analysis: from the chart 2, we can see that the maximum of the light intensity is quite high. However, the distribution of the light is very uneven, which leads to the small illuminated area in

(2) Cellular configuration

The principle of analysis is the same as Uniform distribution.

We got the theoretically optimal coordinates of the eight sets of lamps with the aid of LINGO. The results are as follows:

1、 $(0.613,0.624)$ 2、 $(6.412,0.623)$ 
3、 $(3.513,2.014)$ 4、 $(3.513,5.012)$

5、 $(0.592,3.498)$ 6、 $(6.398,3.502)$

7、 $(0.592,6.374)$ 8、 $(6.412,6.812)$

The best theoretical average illuminance: [LX] 508.143

Using DIALux to simulate: The layout need to be fine adjusted in the DIALux simulation because of some differences between the real lamps and the idealized pointolites in the analog computation of MATLAB and LINGO, and also because the reflection of walls and desks is taken into consideration in the simulation of DIALux.

Using Dialux simulation results of cellular configuration as shown in Fig.3

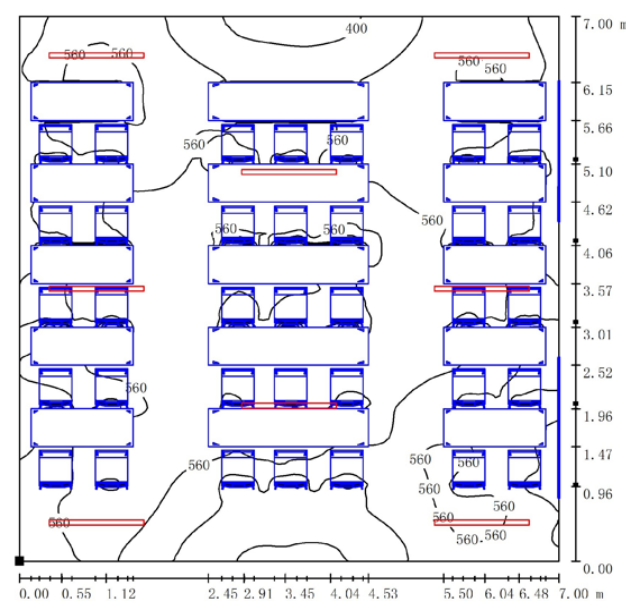

Fig.3 Simulation results of cellular configuration

(3) Elliptic type configuration

The theoretical optimal coordinates of the 8 sets of lamps are as follows:

1、 $(0.111,0.347)$ 2、 $(1.983,1.753)$

3、 $(1.989,5.260)$ 4、 $(3.513,0.112)$

5、 $(3.492,6.798)$ 6、 $(5.098,1.750)$

7、 $(5.192,5.254)$ 8、 $(6.812,3.512)$

The principle of analysis is the same as Uniform distribution.

The best theoretical average illuminance is [LX] 541.

Similarly, using DIALux to simulate:

The layout need to be fine adjusted in the DIALux simulation because of some differences between the real lamps and the idealized pointolites in the analog computation of MATLAB and LINGO. The result is figured out after constant trials and explorations.

Using Dialux simulation results of elliptic type configuration as shown in Fig.4

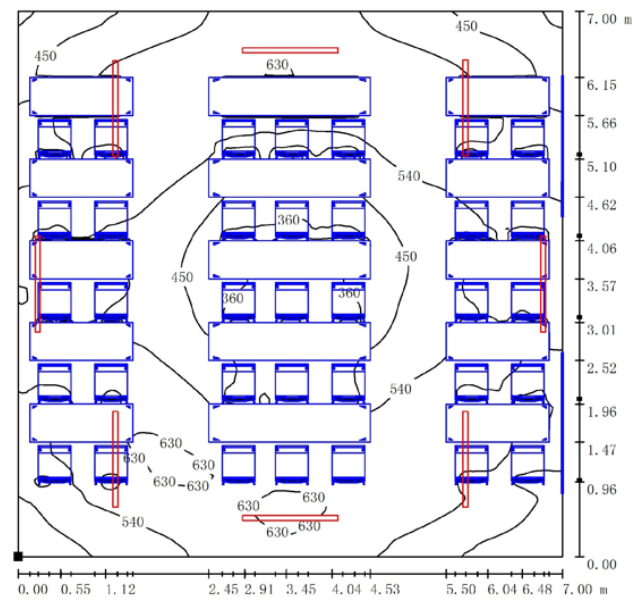

Fig.4 Simulation results of elliptic type configuration 


\section{2) The results of the simulation analysis}

The results of the simulation experiment on the 3 schemes are shown in the following table 2 .

\begin{tabular}{c|c|c|c}
\hline Arrangement of lamps & $\begin{array}{c}\text { The average intensity of } \\
\text { illumination (LX) }\end{array}$ & $\begin{array}{c}\text { Minimum illumination } \\
(\text { LX) }\end{array}$ & $\begin{array}{c}\text { Maximum illumination } \\
\text { (LX) }\end{array}$ \\
\hline Ordinary type & 586 & 207 & 944 \\
Cellular type & 506 & 315 & 627 \\
Elliptic type & 528 & 280 & 706 \\
\hline
\end{tabular}

We can find that every configuration mode meets the standard that the average illumination of CIE is no less than 500LX, but the gap between the minimum illumination and the maximum illumination of the average configuration mode is the biggest, and the evenness degree is the lowest. Compared with the other two kinds, the effective area of this kind of configuration mode is the smallest, so it's quite difficult to make the maximum utilization of the electricity to illuminate the classroom. Among these three kinds of configuration modes, the minimum illumination of the cellular layout is the biggest, for it is 1.52 times as big as that of the uniform layout and it is also 1.13 times as big as that of the elliptical layout. Besides, for the cellular layout, the evenness degree of the illumination, which is the highest and far above that of the uniform layout, reaches 0.622. According to the comparison of so many data of the configuration modes, the cellular layout is the best one.

Moreover, if we describe the number of people which single classroom can contain as the carrying capacity, we can find that the carrying capacity of single classroom will be increased by $20 \%$ on the condition that the existing uniform layout is replaced by the cellular layout. In other words, if the number of the students who study themselves is same, the demand of classrooms can be reduced by $16.7 \%$, and as a result, a great amount of the electricity can be saved.

\section{Conclusion}

Colleges and universities is the densely populated area where teaching, scientific research and living are tightly connected. However, it's also becomes the reason why a great quantity of energy is inevitably consumed here. Under this background, to accomplish the work of energy conservation can not only save money for the school, but also cultivate students' awareness and cognition of energy saving. Even more, it can facilitate the form of resources-efficient society and lay a solid foundation for the sustainable development of China. In view of the energy-wasting problem caused by the classroom illumination, this research group started with the study of the electricity consumption. By interpolation analysis and data fitting with the aid of MATLAB and LINGO, and adoption of the software for lighting design, DIALux, we carried on the simulation test on the classroom light. The comparison among the evenly distributed arrangement, the cellular arrangement and the elliptic type arrangement shows that in the case of meeting the international CIE standard, the cellular arrangement increases the effective lighting area in the classroom and raises the power efficiency of lighting. The result shows that it can truly bring the considerable social benefits and economic benefits, which worth spreading and applying to the practical field.

\section{Reference}

[1]H.J.Zhao:Intemet Fortune,(2009)No.4,p.30-31

[2]Ministry of Construction P.R.China. Standard for lighting design of buildings (GB-50034-2004),(2004)

[3]Gary Steffy:Architectural Lighting Design:China Machine Press,H.L.Rong,L.Li,J.T,Du,

[4]X.Huang:Light Design of Factory Building Based on DIALux Simulation Analysis,China Illuminating Engineering Journal,(2013)No.6,p.120-124

[5]Y.Fan,L.Pei:Electrotechnical Application,(2014)No.2,p.25-32 